\title{
The Effect of Different Lane Occupancy on Road Traffic Capacity
}

\author{
Qiao-Xu Qin ${ }^{1} \&$ Yuan-Biao Zhang ${ }^{1,2}$ \\ ${ }^{1}$ Innovation Practice Base of Mathematical Modeling, Electrical and Information College of Jinan University, \\ Zhuhai, China \\ ${ }^{2}$ Key Laboratory of Product Packaging and Logistics of Guangdong Higher Education Institutes, Jinan \\ University, Zhuhai, China \\ Correspondence: Yuan-Biao Zhang, Innovation Practice Base of Mathematical Modeling, Electrical and \\ Information College of Jinan University, Zhuhai, China. E-mail: zybt@jnu.edu.cn
}

Received: September 25, 2018

Accepted: October 25, 2018 Online Published: October 27, 2018

doi:10.5539/emr.v7n2p54

URL: http://doi.org/10.5539/emr.v7n2p54

\begin{abstract}
In this paper, the data of video is extracted and processed, and the change process of road capacity during the accident is described by using the fuzzy comprehensive evaluation method. By means of cellular automata, the change of road capacity when different lanes are blocked is simulated, and the influence of different lanes on road capacity is analyzed. Then the formula of vehicle queue length based on mathematical relation is established.
\end{abstract}

Keywords: road capacity, cauchy distribution, fuzzy comprehensive evaluation of cellular automata

\section{Introductions}

\subsection{The Related Background of the Problem}

Road capacity refers to the capacity of road facilities to ease traffic flow.That is, the capacity of road facilities to pass traffic flow points within a certain period of time and under normal road, traffic, control and operation quality requirements.Capacity is generally expressed in terms of veh/h (vehicle/hour) and $\mathrm{pcu} / \mathrm{h}$ (equivalent standard small passenger car/hour), and the basic unit is $\mathrm{pcu} / \mathrm{h} / \mathrm{ln}$ (equivalent standard small passenger $\mathrm{car} /$ hour/lane). Capacity is essentially a measure of road load performance, which reflects not only the maximum capacity of the road to unclog traffic, but also the limit value of vehicle operation that the road can bear under the precondition of specified characteristics.

Lane occupation refers to the phenomenon that the capacity of a lane or road cross section decreases in unit time due to traffic accident, roadside parking, occupation and other factors. As the urban road has the characteristics of high traffic density and strong continuity, one lane is occupied, which may reduce the capacity of all lanes of the road section. Even if the time is short, it may also cause vehicle queuing and traffic obstruction.If mishandled, even appear regional congestion.

When the actual traffic volume on the road is less than its capacity, the driving vehicles on the road are in a free driving state, with higher speed and less traffic density. When the actual traffic volume on the road is close to or equal to its capacity, the vehicles on the road are tracked with the speed approaching uniform, resulting in the phenomenon of fleet driving, and the headway distribution is close to the average value. When the actual traffic volume on the road exceeds its capacity, the density of vehicles on the road increases and the speed decreases, resulting in traffic congestion and obstruction. The main factors affecting road capacity are road conditions, traffic conditions and external environment.

\subsection{Research Design}

Firstly, the traffic flow on the exit road was calculated according to the surveillance video from 16:38-17:03 of a certain place in China, and the change process of the actual capacity of cross section of the accident between the occurrence and evacuation was described. In order to describe the process of capacity change, it is necessary to find out the factors affecting capacity and determine an evaluation index of capacity. Thus describes the change process of road capacity in traffic jams.

Then according to the conclusions of the first part, the differences of the traffic accident in the same cross-section on the actual capacity of the cross-section are analyzed. According to the accident statistics of 
vehicles on the road in a certain place in China when one or two lanes are occupied between 17:38-18:03. In order to explain the difference in the influence of different lanes occupied by the same cross-sectional traffic accident on the actual capacity of the cross-sectional traffic accident, it is necessary to analyze the difference in the capacity of the lane occupied in the process of occupying two or three lanes and one or two lanes. Considering that the situation may have some contingency, it is also necessary to simulate the traffic jam situation, and simulate the impact of two kinds of congestion on the actual traffic capacity of the road under general circumstances.

Finally, a mathematical model was constructed to analyze the relationship between the length of vehicle queue on road sections affected by traffic accidents and the actual capacity, the duration of accidents and the upstream traffic flow of road sections. Considering that when two lanes are blocked, the traffic volume of the other lane can be used to measure the actual traffic capacity of the road surface. The duration of the accident has an impact on the queue length and traffic volume. Therefore, a mathematical model is built to measure the relationship between the four indexes by considering the relationship between the four indexes.

\section{The Establishment and Solution of the Model}

\subsection{Evaluation of Road Capacity}

According to the data, speed, traffic flow, abnormal travel time and queuing length have the greatest impact on the measurement of road traffic capacity. Therefore, we choose these four indicators to measure the road traffic capacity. This paper use the fuzzy comprehensive evaluation method to evaluate this.

First, the evaluation weight needs to be determined.Set $A=\{a 1, a 2, a 3\}$;The corresponding weight set $\mathrm{B}=\{\mathrm{b} 1, \mathrm{~b} 2, \mathrm{~b} 3\}$; In order to determine the weight set, it is advisable to set the strength set as \{strong, strong, strong, slightly strong, general $\}$, and the corresponding values are $5,4,3,2,1$, respectively. It can be considered that it is most suitable to take the biased large cauchy distribution function as the membership function of the evaluation.

The larger cauchy distribution function is as follows:

$$
f(x)=\left\{\begin{array}{l}
\frac{1}{1+a(x-b)^{-2}}, 1 \leq x \leq 3 \\
c \ln x+d, 3 \leq x \leq 5
\end{array}\right.
$$

Where $\mathrm{a}, \mathrm{b}, \mathrm{c}$ and $\mathrm{d}$ are undetermined coefficients.In fact, the strength of "strong" is the membership degree of 1 , when the intensity is "strong" membership degree is 0.6 , when the intensity is "general", membership degree is 0.1 , thus determine the undetermined coefficient values of $\mathrm{a}, \mathrm{b}, \mathrm{c}, \mathrm{d} \mathrm{a}=0.1239$, respectively, $\mathrm{b}=2.3713, \mathrm{c}=$ $0.3915, d=0.3699$, the coefficient of four generation into the equation to get the membership of each factor and normalized values in the following table.

Table 1. Membership value and normalization value of each factor

\begin{tabular}{lllll}
\hline Indicators & Speed & traffic & Abnormal travel time & Queue length \\
\hline Give value & strong & strong & A little stronger & A little stronger \\
Membership values & 0.6 & 0.6 & 0.4249 & 0.4249 \\
The normalized value & 0.2927 & 0.2927 & 0.2073 & 0.2073 \\
\hline
\end{tabular}

So the weight vector we get is $(0.2927,0.2927,02073,0.2073)$.

The score of road capacity was calculated according to the weight, and the broken line chart of road traffic capacity between the occurrence of traffic accidents and the evacuation period was drawn with Matlab. The results are shown in the following figure: 


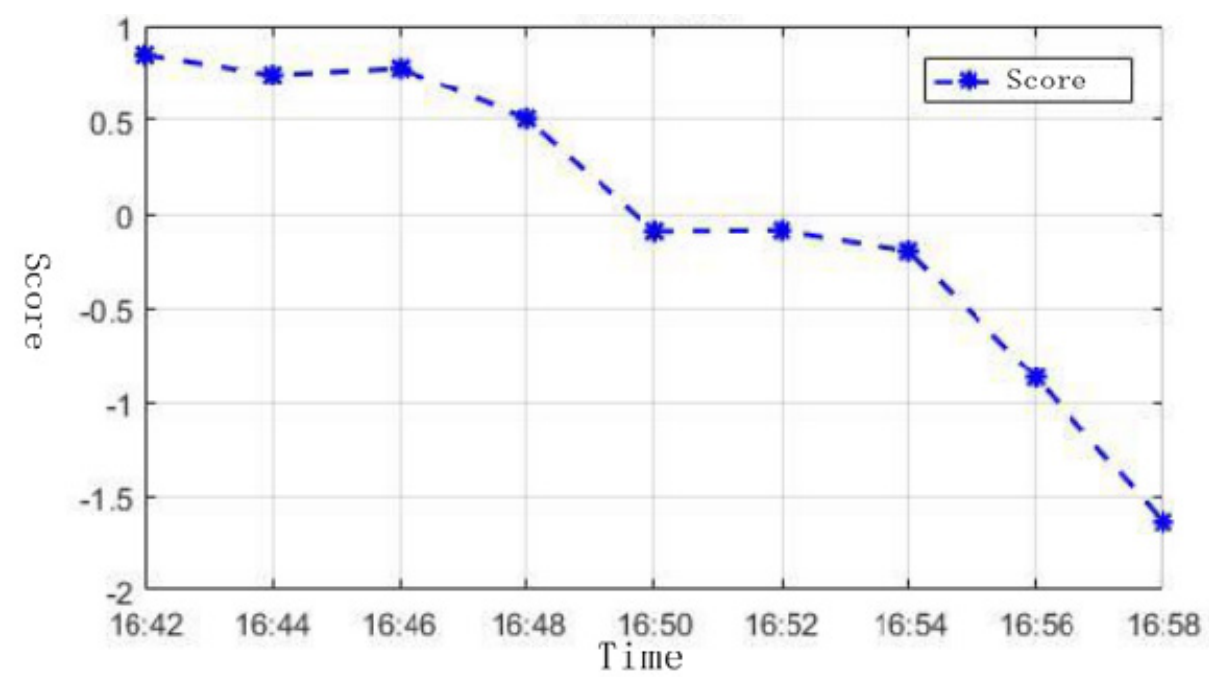

Figure 1. Changes in road capacity over time

The line chart shows that just happened in traffic accidents, road traffic capacity will be a certain degree of decline, but the degree of decline is not large, then there will be a small rise, this may be due to the upstream intersection traffic lights time just blocked the arrival of the next batch of vehicles, makes the accident place have enough time to digest has blocked vehicles, so the transport capacity will have certain up.There are two phases in the chart where capacity is relatively stable, probably for all of these reasons. However, with the arrival of vehicles accumulated upstream and the arrival of vehicles already blocked, there will be more serious congestion, and the capacity of roads will decline significantly.

\subsection{Analysis of the Causes of Congestion}

Through observation and analysis, this paper thinks that the factors that have a greater impact on road congestion include the number of vehicles at the entrance of the community, the number of large and small cars on the road, the relatively serious traffic congestion and the congestion digestion caused by upstream traffic lights. The influence degree of these factors is analyzed one by one.

(1) Number of large vehicles

Combined with the actual situation, it can be seen that large vehicles on the road, such as buses and buses, need to change lanes when driving to the place where the accident occurs. However, these large vehicles often require more time and take up more lanes due to their large size, so they will have some impact on congestion.

Similar to the above question, the number of large vehicles passing the accident cross section during the accident occurrence is first calculated, and then the correlation test is conducted by using SPSS statistical software. The statistical values and test results are as follows:

Table 2. The number of large vehicles on the accident cross section

\begin{tabular}{llllllllll}
\hline Time & $16: 42$ & $16: 44$ & $16: 46$ & $16: 48$ & $16: 50$ & $16: 52$ & $16: 54$ & $16: 56$ & $16: 58$ \\
\hline Large vehicles & 7 & 1 & 1 & 1 & 1 & 2 & 2 & 3 & 1 \\
\hline
\end{tabular}

The significance test results were 0.275 , greater than 0.05 , the original hypothesis was rejected, indicating that there was no significant correlation between the two. Therefore, the number of small and large cars will have a certain impact on the road capacity, but not much.

(2) The occurrence of severe congestion

After the accident.There is only one lane left for the road to pass. When the traffic flow is relatively small and there is no car to supplement later, the single lane will have enough time for vehicles to pass. However, once the traffic flow increases, it will lead to more and more queues and more vehicles on the road cannot be digested, which will lead to traffic congestion. Through observation, several times of obvious congestion in the traffic jam 
stage were calculated and analyzed.

The first congestion occurred between 16:42 and 16:47.During the period from 16:42 to 16:44, the actual capacity increased with the passing of large vehicles. However, the cross-section of the accident was always in the vehicle queue due to the continuous supplement of the upstream traffic, so the actual capacity was not significantly improved.In the period from 16:44 to $16: 47$, the upstream traffic flow decreases, and the accident cross section is not fully saturated by vehicles in part of the time. Therefore, the road cross section of the downstream congestion has enough time to digest the accumulated vehicles, so the road capacity will increase slightly.

The second traffic jam is between 16:47 and 16:50, and there is a certain amount of traffic flow in the upstream between 16:47 and 16:49, but no large vehicles appear. Therefore, the queue length of vehicles is small and evacuation speed is fast, so the actual capacity will decline to some extent, but the decrease is not obvious. Within 16:49 to $16: 50$, there are still no more large vehicles, but the traffic flow is obviously increased, so the road capacity will have a more obvious decline at this time.

The third traffic jam is between 16:50 and 16:53. During this period, there will be continuous traffic coming from the upper and middle reaches, but the traffic flow is relatively stable without sudden increase or decrease. Therefore, the traffic volume of this period is relatively stable and maintained at a relatively low level.

Traffic accident 16:54 $\sim$ 16:58, at this stage, the cross section of congestion exists still undigested vehicles, while the upstream flying much road vehicles, which also contains more big cars, and mouth from the village of vehicles will also increase, various factors will strengthen the congestion level of the traffic jam on cross section, road traffic capacity at this time there will be a very significant decline.

(3) The influence of traffic lights at the upstream junction

The traffic lights at the upstream junction determine whether the upstream vehicles reach the downstream within a certain period of time, and the upstream vehicles largely determine the congestion on the cross section. Therefore, the traffic light on the road has an influence on the traffic volume of the accident cross section to some extent.

When there are fewer vehicles in the accident cross section and the upstream traffic light just stops the arrival of vehicles, there are few vehicles on the road and the traffic capacity is good.

When there are fewer vehicles at the accident cross section and more vehicles coming from upstream, there is enough time on the road to pass through these vehicles, and the capacity is better.

When there is a certain number of vehicles at the accident cross section, but there are fewer vehicles coming upstream, the congestion area has enough time to digest these vehicles, so the road capacity is better.

When there are a certain number of vehicles at the cross section of the accident and more vehicles are driven from the upstream, the traffic volume will be greatly affected. As only one lane of the road is feasible, the traffic volume is much smaller than the accumulation volume of vehicles, which will cause more serious congestion.

\section{The Influence of Different Occupied Lanes on Road Traffic Capacity}

\subsection{Description of Changes in Road Capacity During Lane 1 and 2 Traffic Jams}

In the same way as the road capacity rating in the first part, the statistical data obtained in video 2 are unified polarity and standardized, and the road capacity rating at each moment between accidents is calculated according to the coefficients of each variable obtained in the first part. Finally, according to the score of each moment, the real-time rating of road capacity when two or three lanes are occupied in video 1 and road capacity when one or two lanes are occupied in video 2 is reflected in a line graph with Matlab, and the differences between the two are analyzed from the line graph. The time on the horizontal axis is characterized by the time after the accident. 


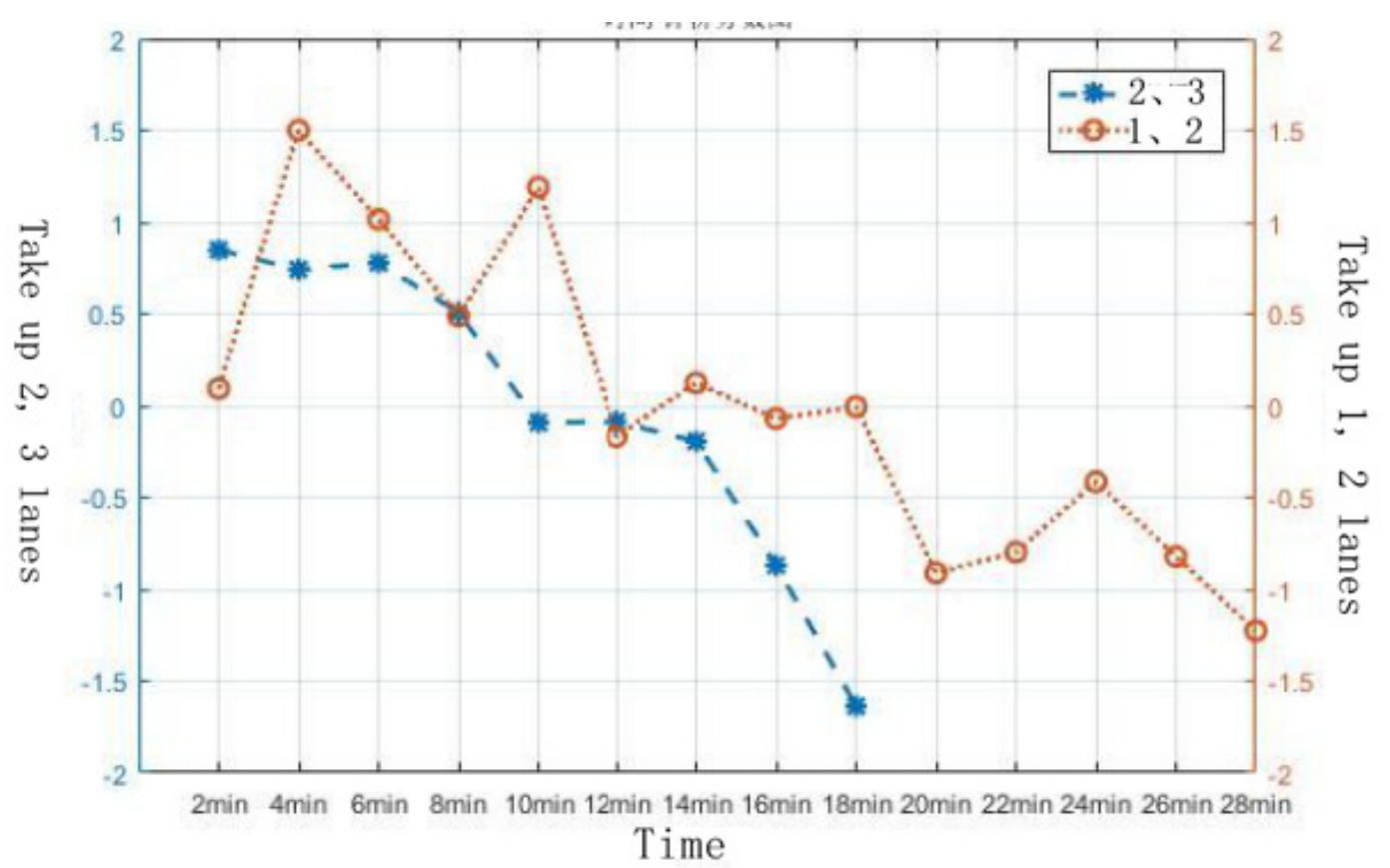

Figure 2. Changes in scores for two types of traffic jams

Can be seen from the chart, most of the time of the accident, a video component when the first and second lanes jam traffic capacity values are higher than the ratings of video one occurs when two or three lanes jam road traffic capacity rating value, on the basis of preliminary judgment of the road can be the same cross-sectional, block when the two or three lanes have a larger impact on road traffic capacity, block 1,2 driveway impact on road traffic capacity is relatively small.

Considering that only the road conditions at the time of two accidents are given in video, which may and will be somewhat accidental, it is necessary to use cellular automata to simulate the congestion situation and find the general rule.

\subsection{Simulation of Cellular Automata}

\subsubsection{Model Preparation}

Cellular automata is a dynamic system that defines a cellular space consisting of cells with discrete and finite states and evolves in discrete time dimension according to certain local rules. In this paper, cellular cells are used to replace the vehicles on the road to simulate the traffic flow on the road. When simulating two vehicles with accidents, only two fixed cellular cells should be placed in the position where the accident occurred to simulate the traffic jam on the road.

(1)Matrix of the rear lanes:

Construct a matrix of $\mathrm{m}^{*} \mathrm{n}\left(81^{*}\right), \mathrm{m}=80$ represents the number of cells contained in each lane, each cellular side length is 4 meters, $n=5$ represents the number of lanes and boundaries, namely: three lanes + two lane boundaries. There are three types of cellular cells in the matrix: cellular cells occupied by vehicles, empty cellular cells and inaccessible cellular cells, which are respectively represented by values 1,0 and -1. Matlab was used to visualize the matrix, and the simulation graph of the road was obtained as follows:

\section{$\ldots$}

The road is upstream on the right and downstream on the left, with traffic moving from right to left.

(2)Type of car:

In this model, it is necessary to consider small vehicles and large vehicles. Small vehicles are general cars, taxis, etc. Large vehicles include buses, medium trucks and other models. Small vehicles are represented by one cell 
generation and large vehicles by two cells.

(3) Update interval

Usually, the reaction time of the driver under normal driving is the updated time step in the model. The response time of the driver is generally $1 \mathrm{~s}$, that is, the update time step is also subtracted $1 \mathrm{~s}$.

(4) The boundary conditions

Generally, there are two boundary conditions of cellular automata model, which are periodic boundary conditions and open boundary conditions. The opening boundary condition means that the vehicle no longer enters the starting point after leaving the end point of the simulated road section. It can be seen from the analysis of the road section of this topic that, because the simulation time is short and traffic accident occurs on the simulated road section, the passing driver will choose to avoid the road section when passing again, and the possibility of entering the road section for the second time by the same car is small, so the open boundary condition is adopted in this model.

\section{(5)Grid model}

Traffic road simulation is a random event, so the time interval for vehicles to arrive at the simulated road section is a random quantity. Theoretically, it follows the poisson distribution principle, that is, the generation of vehicles has the following characteristics:

The vehicles are produced without hysteresis, that is, in the time interval without overlapping, the vehicles are produced independently.

For sufficiently small $\Delta t$, the probability that a car is produced within the interval $|t, \Delta t|$ is independent of $t$, but is proportional to $\Delta t$, and the vehicle is produced stably.

For sufficiently small $\Delta t$, the probability of two or more vehicles in one lane within the interval $[t, \Delta t]$ is very small, and the occurrence of vehicles is universal.

(6) Rules for vehicle advance and lane change

The forward rule: if the state of position $i$ at time $t$ is car and position $i+1$ is empty, then the state of position $i+1$ at time $\mathrm{t}+1$ is empty and position $\mathrm{i}+1$ is car; $\mathrm{I}$ is the vehicle number

Lane change rule: if both positions $i$ and $i+1$ are cars at time $t$, the probability of car trying to change lanes is the same as that of right.

\section{(7) Speed setting}

According to the 1st video, in the case of unblocked road, the advance speed of the vehicle is $9.86 \mathrm{~m} / \mathrm{s}$, so the average $\mathrm{v}$ is set to be equal to $2 \mathrm{cell} / \mathrm{s}$, which is equal to $9.8 \mathrm{~m} / \mathrm{s} . \mathrm{V}=3$ largest cell $/ \mathrm{s}=14.7 \mathrm{~m} / \mathrm{s}$

\section{(8) Improved progressive rule}

Find the cell number(i) between the current position of the car and the nearest obstacle. Number (i) refers to the number of cells between the car and the obstacle.

\section{(9) Speed Up}

Any vehicle $\mathrm{i}$ at the current moment, when number $(i)>v(\text { now })^{*} t$ means that the road ahead is unobstructed. According to the daily phenomenon, the driver will tend to accelerate, thus generating random Numbers between 0 and 1 . If it is less than $\mathrm{p}=0.8$, then $v($ next $)=\min (v($ now $+1, v($ mas) $))$. The number (i) represents the number of cells between the car and the obstacle.

(10)Collision avoidance

For any vehicle $\mathrm{i}$ at the current time, when number $(i)>v(\text { now })^{*} t$, in order to avoid collision, the deceleration should not be too large at the same time. If $\mathrm{P}(r d)=1$, then $v($ next $)=v(i)-1$

(11)Random slowdown

Drivers may often slow down due to non-traffic factors, which will have a certain impact on traffic, but the probability of random deceleration is small, so $\mathrm{P}(r n d)=0.3, v($ next $)=v(i)-1 . v(n e x t)$ is the speed of the vehicle at the next moment, $v(i)$ is the speed of the ith vehicle.

(12) Improved lane changing rules

It often takes a certain amount of time to change lanes in crowded situations, so we extend the time of changing lanes in the model. 


\subsubsection{Simulation of the Model}

Matlab is used to simulate the dynamic process of lane occupation with the help of cellular automata rules (see annex 1 for matlab code). The simulation time is set to 20 minutes, and the accident in the second or third lane is regarded as case one, and the accident in the first or second lane is regarded as case two. Screenshots of traffic on the road are taken every five minutes and analyzed. Matlab was used to visualize the matrix, and the simulation graph of the road was obtained as follows:

The following figure shows the simulation of the driving condition of the road at $300 \mathrm{~s}, 600 \mathrm{~s}$, 700s and $900 \mathrm{~s}$ when the road is blocked on the second and third lanes (the road is upstream on the right and downstream on the left, with traffic moving from right to left).

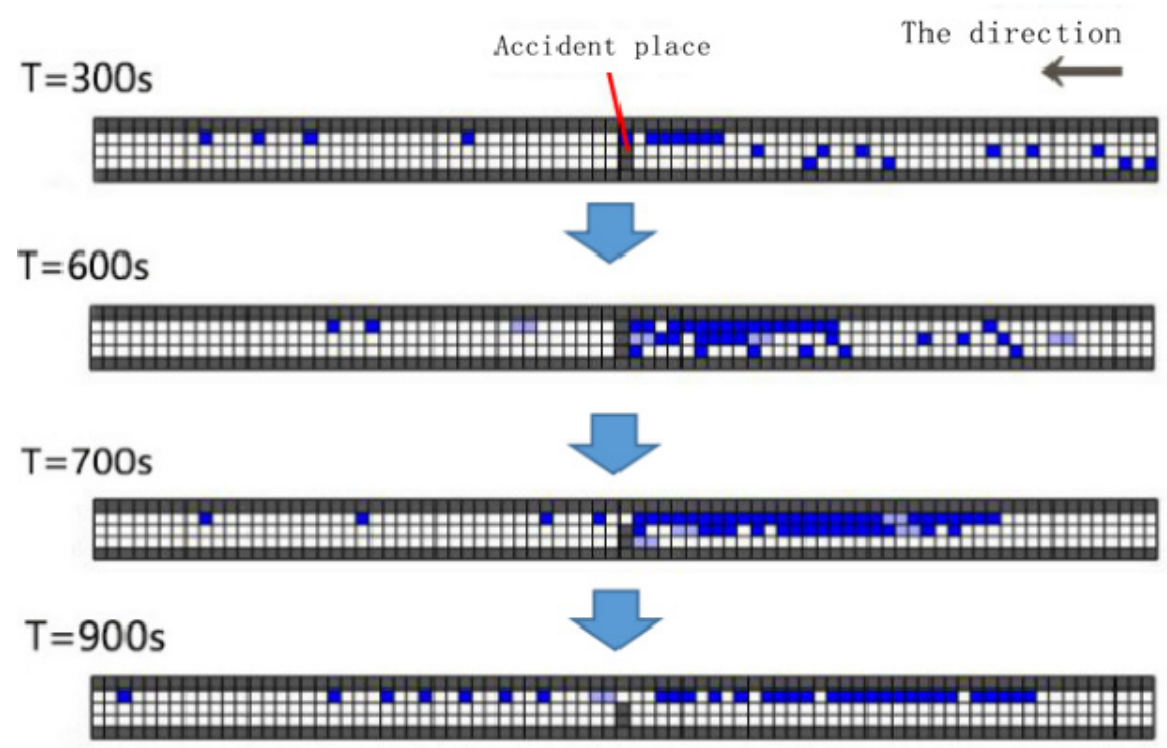

The following four pictures show the traffic condition simulation of the road at 300s, 600s, 700s and 900s when the two lanes are blocked (the road is upstream on the right and downstream on the left, with traffic moving from right to left).

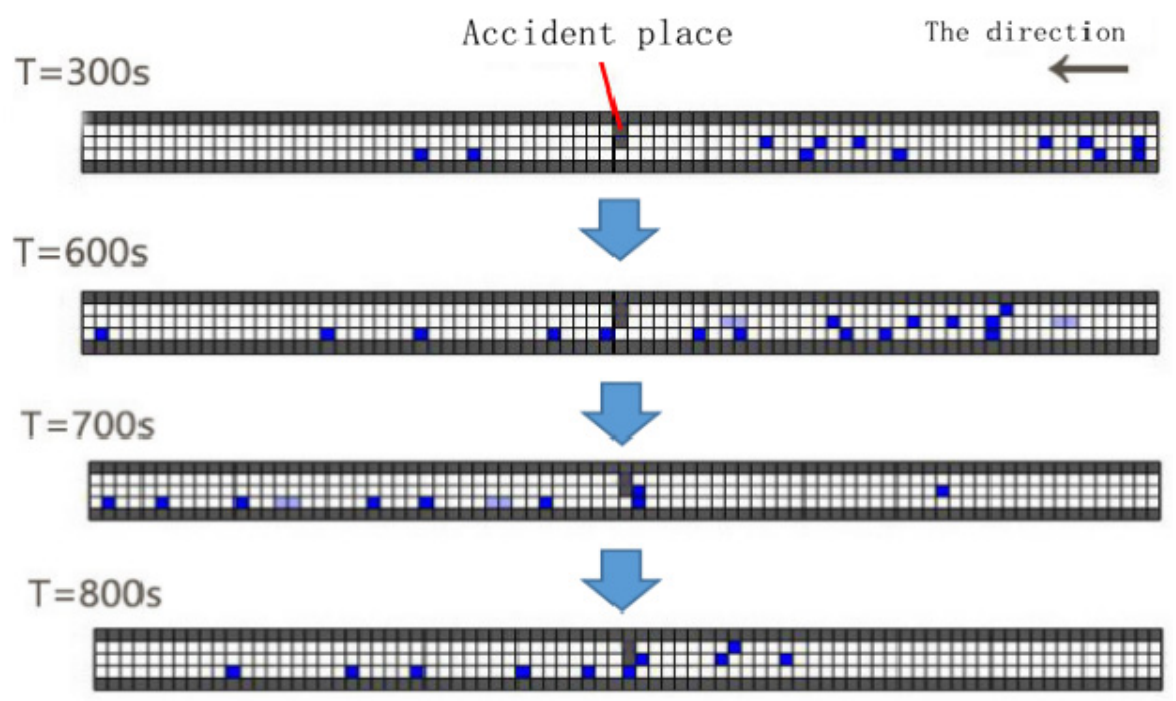

First of all, from the comparison of two traffic jams, it can be seen that the traffic jams on the first and second lanes are much better than those on the second and third lanes. The road is basically a vehicle that can digest the traffic while blocking one or two lanes, thus keeping the road smooth. The reason may be that the speed of driving in different lanes has a great impact. According to the regulations of China's traffic laws and people's driving habits, the driving speed of outer lane, middle lane and inner lane increases successively. Through 
observation and analysis of two video's, it can be found that the driving speed of lane 1 is much less than that of lane 2 and 3, and lane 3 is faster than lane 2. So the average speed in video two is greater than video one.

Then, through the simulation of cellular automata, the difference of road capacity is illustrated from specific data.Through multiple measurements and averaging, it was found that the average time required for vehicles to pass this section of road when blocking the second and third lanes was $121 \mathrm{~s}$, the traffic flow was $110.5 \mathrm{pcu} / \mathrm{min}$, and the average time required for vehicles to pass this section of road when blocking the first and second lanes was $47 \mathrm{~s}$, and the traffic flow was $296 \mathrm{pcu} / \mathrm{min}$. It is obvious from the specific data that the road capacity is better when one or two lanes are blocked.

\section{Factors Affecting Vehicle Queue Length}

\subsection{Ideas of the Model}

The road is divided into three lanes. When two lanes are occupied, only one lane can be used for vehicles. According to the reference, the road capacity can be simply measured by the traffic flow. Passing vehicles reduce the length of the queue when stuck. On the other hand, vehicles coming from the upstream junction, vehicles coming from the residential junction and vehicles not yet in use will increase the queue length. The number of vehicles coming is closely related to the number of traffic jams. The length of the queue is equal to the product of the traffic flow per unit time, time and the yard. Considering the difference between large and small cars, standard traffic is used to eliminate this effect.

To sum up, the length of queue is divided by the number of lanes and the length of queue of existing vehicles by the increased length of vehicles in the upstream and in the community minus the length of vehicles that have passed divided by the number of lanes. The increase of traffic length on the upper and small intersections is the ratio of traffic flow, time and length. The reduced length is the product of capacity, time and length. By integrating the model, the relationship between queue length and actual capacity of accident cross section, accident duration and upstream traffic flow can be obtained.

\subsection{Model Establishment}

From the above analysis, it can be obtained

$$
L_{\text {queue }}=\frac{1}{N_{\text {Lanes }}}\left(L_{u p}+L_{\text {village }}-L_{c}\right)+L_{0}
$$

$L_{\text {queue }}$ is the queue length at a certain moment, $N_{\text {Lanes }}$ is the number of lanes, $L_{u p}$ is the length of the increase of upstream vehicles, $L_{\text {village }}$ is the length of the increase of vehicles in the community, $L_{c}$ is the length of the decrease of tolls, $L_{0}$ is the existing queue length.

Specific to the impact of each variable:

$$
\left\{\begin{array}{l}
L_{u p}=\int V F_{u p} \mathrm{~d} t \cdot \text { Length } \\
L_{\text {village }}=\int V F_{\text {village }} \mathrm{d} t \cdot \text { Length } \\
L_{c}=\int C \mathrm{~d} t \cdot \text { Length }
\end{array}\right.
$$

$L_{u p}$ is the length of the increase of upstream vehicles, $V F_{u p}$ is the upstream traffic flow, Length is the length of the standard vehicle, $L_{\text {village }}$ is the length of the increase of the district vehicles, $V F_{\text {village }}$ is the length of the district traffic flow, $L_{c}$ is the length of the decrease of traffic flow, $C$ is the actual capacity.

To sum up, the queue length at a certain moment is:

$$
L_{\text {queue }}=\frac{1}{N_{\text {Lanes }}}\left[\int\left(V F_{\text {up }}+V F_{\text {village }}-C\right) \mathrm{d} t \cdot \text { Length }\right]+L_{0}
$$

$L_{\text {queue }}$ is the queue length at a certain time, $N_{\text {Lanes }}$ is the number of lanes, $V F_{u p}$ is the upstream traffic flow, $V F_{\text {village }}$ is the plot traffic flow, $C$ is the actual capacity, Length is the standard vehicle length, $L_{0}$ is the queue length of existing vehicles.

In this formula, $t$ is the duration of the accident. From the actual situation, the queue of vehicles in traffic jam does not always reach the upstream junction, so it takes a certain amount of time for vehicles coming from the 
upstream to travel to the queue and stop. Assume that the upstream stop vehicles from crossing to the team for the uniform all the way, the consumption of the time for $t_{v}=\frac{s-L_{\text {quene }}}{v}, s$ for the accident site, the distance to the upstream intersection queue length $L_{\text {queue }}$ for some time, $\mathrm{v}$ is the average vehicle speed, the actual time for $t_{\text {true }}=$ $\left(t-t_{v}\right)$, for the same reasons, village vehicles also consume a certain amount of time into the team, but district intersection between vehicle team, vehicle a very short period of time in the team, can think this behavior is "even", is not time-consuming, so only need to consider the upstream vehicles enter the team takes time. When the consumption time is substituted into formula (1), the total formula is:

$$
L_{\text {queue }}=\frac{1}{N_{\text {Lanes }}}\left\{\left[\int V F_{\text {up }} \mathrm{d}\left(t-\frac{s-L_{\text {queue }}}{v}\right)+\int\left(V F_{\text {village }}-C\right) \mathrm{d} t\right] \cdot \text { Length }\right\}+L_{0}
$$

$L_{\text {queue }}$ is the queue length at a certain time, $V F_{u p}$ is the upstream traffic flow, $t$ is the duration of the accident, $s$ is the distance from the accident occurrence site to the upstream junction, $v$ is the average vehicle speed, $V F_{v i l l a g e}$ is the community traffic flow, $C$ is the actual capacity, Length is the standard vehicle length, $L_{0}$ is the queue length of existing vehicles.

\subsection{Model Test}

The effect of the relation formula of queue length derived above was tested, and the duration of road congestion, road capacity, upstream traffic flow and regional traffic flow in the period of 16:43:42-16:44:02 in video 1 were selected for statistical analysis. The statistical results are as follows:

Table 3. Traffic indicators for 2 and 3 lane occupancy

\begin{tabular}{lc}
\hline Indicators & Value \\
\hline Time of duration $(\mathrm{h})$ & 0.0083 \\
Actual capacity $(\mathrm{pcu} / \mathrm{h})$ & 1005 \\
Average upstream traffic flow $(\mathrm{pcu} / \mathrm{h})$ & 780 \\
Average traffic flow at small intersections $(\mathrm{pcu} / \mathrm{h})$ & 96 \\
\hline
\end{tabular}

To facilitate comparison, this paper defines an ideal queue length. The queue length is difficult to determine due to the different captains in the three lanes. Therefore, the vehicles at the end of a longer queue are added to a shorter queue, which makes the queue length of three lanes equal, and the leader at this time is defined as the ideal queue length.

Substitute each value into formula (2) to calculate the queue length.In the ideal queuing state, the queue length at 16:44:02 is 5.354 meters less than that at 16:43:42. It can be seen from the data that the length of 5.354 meters is equal to that of an ordinary car plus the gap between the front and the back of the car. After the actual queuing situation at 16:43:42 and 16:44:02 is transformed into the ideal queuing situation, the visualization is carried out in Matlab. The results are shown as follows:

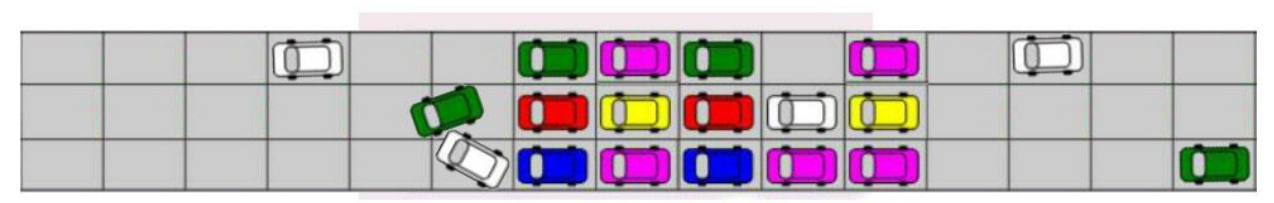

The queue at 16:43:42

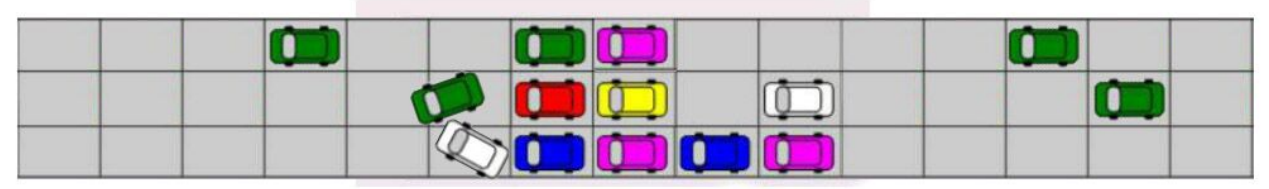

The queue at 16:44:02 
After transforming the actual queuing situation into the ideal queuing state, it can be seen that the length of one row of cars is longer at the first time than at the second time. The gap between two rows of cars plus the front and back is equal to 5.354 meters, so it can be known that the estimation effect of the model is relatively good.

\section{Evaluation and Improvement of the Model}

\subsection{Advantages of the Model}

(1) From the speed, the number of cars, each car due to time delays caused by traffic jams and traffic jam when the Angle of vehicle queue length four evaluates the capacity of the road, but also consider the mouth area vehicle number, the number of large cars and small cars on the road, more severe upstream traffic congestion and congestion caused by digestive four actual situation, make the evaluation more comprehensive.

(2) The actual situation of the road in a period of time was simulated with cellular automata, and the actual changes of the vehicles on the road were simulated, making the evaluation results more realistic and more consistent with the actual situation.

(3) When estimating vehicle queue length, the mathematical relation of queuing is directly considered from the perspective of actual situation, rather than using simulation or statistical principle to estimate, and the result is more accurate and more consistent with the actual situation.

\subsection{Disadvantages of the Model}

(1) The required data is mainly obtained by observing video statistics, and the results may have errors.

(2) The rule setting of cellular automata model is limited to the information in video, which makes the prediction interval not accurate enough. If there are conditions, more sections can be simulated and parameters can be optimized to make the prediction results more accurate. Or we can use the combination of queuing theory and cellular automata to consider the vehicle queuing problem from the mathematical and practical aspects.

\section{References}

BaiduBaiKe: Road capacity Retrieved from https://baike.baidu.com/item/\%E9\%81\%93\%E8\%B7\%AF\% E9\%80\%9A $\%$ E8\%A $1 \% 8 \mathrm{C} \% \mathrm{E} 8 \% 83 \% \mathrm{BD} \% \mathrm{E} 5 \% 8 \mathrm{~A} \% 9 \mathrm{~B}$

Han, Z. G. (2014). Mathematical modeling method and its application. Beijing advanced education press.

Highway capacity manual. https://wenku.baidu.com/view/de2a1e8acc22bcd126ff0cc0.html?pn=1

Juan, X. (2014). Research on single-lane traffic flow model based on cellular automata. Chang 'an University.

Law of the People's Republic of China on road traffic safety. Retrieved from https://baike.baidu. com/item/\%E4\%B8\%AD\%E5\%8D \%8E \%E4\%BA\%BA\%E6\%B0\%91\%E5\%85\%B1\%E5\%92\%8C $\%$ E5\%9 B\%BD $\%$ E9\%81\%93\%E8\%B7\%AF\%E4\%BA\%A4\%E9\%80\%9A\%E5\%AE\%89\%E5\%85\%A8\%Е6\%B3\% $95 / 149277 ? \mathrm{fr}=$ aladdin

Long, X. (2012). Comparative analysis and optimization of vehicle tracking model for emission measurement. Beijing jiaotong university.

Nai-Shuo,T. (2008). Discrete time queuing theory. Beijing: Science Press.

Qi, P. Y., \& Xiao, Q. L. (2000). Traffic flow model under the control of signal lights. Journal of Xi 'An Jiaotong University, 35(3), 301-305.

Zhong, B. G. (2014). Optimization of models for emission measurement. Beijing Jiaotong University.

\section{Copyrights}

Copyright for this article is retained by the author(s), with first publication rights granted to the journal.

This is an open-access article distributed under the terms and conditions of the Creative Commons Attribution license (http://creativecommons.org/licenses/by/4.0/). 